\title{
Parents' Experiences of Support in NICU Single-Family Rooms
}

\author{
Lisa Xiaoyang Liu, $M S_{c}(A)$ \\ Maryam Mozafarinia, RN, MSc \\ Anna Axelin, RN, PhD \\ Nancy Feeley, RN, PhD
}

\section{Disclosure}

The authors have no relevant financial interest or affiliation with any commercial interests related to the subjects discussed within this article. No commercial support or sponsorship was provided for this educational activity.

\begin{abstract}
Purpose: This study aimed to explore support for mothers and fathers in single-family rooms (SFRs) of a NICU.

Design: A qualitative descriptive design was employed.

Sample: A convenience sample of 15 parents (nine mothers, six fathers) were recruited from a Level III NICU.

Method: During their infants' hospitalization, each parent recorded their thoughts and feelings regarding support whenever appropriate over a period of 48 hours using Handy Application to Promote Preterm infant happY-life (HAPPY), an android recording application.

Results: Parents felt supported when staff facilitated their learning in a collaborative manner, fostered their optimism, and provided situational assistance. Continuity and consistency of care and presence were important characteristics of supportive nursing care. Though SFRs offered privacy for parents to learn and to be with their infants, the design limited parental access to nursing and medical staff, which sometimes prevented parents from receiving adequate support and partaking in decision making concerning their infants' care.
\end{abstract}

Keywords: single-family room; NICU design; support; access to staff; isolation; parents

$\mathrm{F}$ OR THE PAST TWO DECADEs, there has been increasing interest in the design of NICUs. ${ }^{1}$ The NICU is a specialized care unit that cares for newborns who require intensive medical attention. Most infants admitted to the NICU are preterm (born before 37 weeks of gestation), have low birth weight, or have medical conditions such as cardiac issues, infections, or birth defects. According to the Centers for Disease Control and Prevention, of the 3.9 million births in the United States in 2016, 8.2 percent were born with birth weight $<5.5 \mathrm{lb}$., and 9.8 percent were preterm. Giving birth to a sick or preterm infant can be unexpected and overwhelming for any parent, and the NICU environment can be a factor that contributes to the emotional well-being of parents and families in the NICU., ${ }^{2,3}$

Traditional open wards (OWs) have historically accommodated $10-50$ beds in one space so that all neonates are visible to the staff. ${ }^{4,5}$ However, crowding of parents, staff, and equipment within OWs generates an environment that exposes the developing neonate to disruptive sensory stimulation and deprives parents of privacy. Informed by seminal evidence that environmental factors such as high levels of noise and bright light can lead to deleterious developmental 
outcomes for infants, ${ }^{6-8}$ the design of traditional NICUs have been called into question. In 1992, the first recommendation was put forth suggesting single-family rooms (SFRs) as the optimal setting in which to care for critically ill and preterm newborns. ${ }^{9}$ Since then, architectural transition toward SFR design has been gradually implemented across North America and worldwide to address limitations of the traditional OW. ${ }^{1,10}$ However, design experts have speculated that a possible drawback of SFR is the relative isolation of families from staff and from other families, which may cause parents to feel less supported. To date, some studies have examined parental satisfaction with SFR NICUs, ${ }^{11-13}$ but few studies exist that examine parents' experience of support within these rooms.

Current evidence regarding the benefits of SFRs is mostly derived from quantitative studies either comparing units of different designs across multiple sites or comparing the same site pre/posttransition from OW to SFR. These studies suggest that when compared to OWs, SFRs have lower noise levels, ${ }^{14,15}$ better lighting control, ${ }^{11,15}$ and provide more privacy for infants and their parents. ${ }^{5,12,15}$ Improved privacy in SFRs promotes breastfeeding and better attachment between parents and infants. ${ }^{10,11,16}$ Furthermore, neonates in SFRs have better medical outcomes, including fewer apnea events for apnea-prone infants, ${ }^{16}$ greater infant weight at discharge,${ }^{17}$ and reduced length of hospital stay. ${ }^{18}$

Experts in the field have also cautioned against potential disadvantages of SFRs, which include isolation of families, ${ }^{19}$ reduced family-to-family interaction, ${ }^{20}$ and impeded access to staff. ${ }^{21}$ Moreover, recent studies have compared parental stress in OW and SFR NICUs with either conflicting results or nonsignificant findings. ${ }^{10,22,23}$ Pineda and colleagues ${ }^{22}$ found increased parental role stress for parents in SFRs, and suggested that this may be due to isolation and inadequate levels of support. Domanico and colleagues ${ }^{11}$ examined family-to-family interaction by surveying parents from SFRs and OWs. Parents in OWs felt they were better able to meet peers than parents in SFRs, but no significant differences were found between the two settings when parents were asked about whether interactions with peers made their hospital stay better. In contrast, Harris and colleagues ${ }^{20}$ observations of parents revealed that parent-to-parent interactions were minimal for both SFR and OW units when parents were at besides. However, parents with infants in OWs did interact with each other in lounges when the NICU was closed for visitation. Currently, no study has investigated support in-depth using a qualitative methodology, and inconsistent findings from quantitative and observational studies demonstrate the need for further research to explore parents' experiences of support in SFRs.

Support is an essential element of the overall hospital experiences for parents whose infant is critically ill. ${ }^{24}$ Miles and colleagues identified the four dimensions of nursing support for parents with hospitalized infants as (1) supportive communication and ongoing information, (2) helping parents maintain their parental role, (3) providing emotional support, and (4) providing expert nursing care to their infants. ${ }^{24}$

Domanico and colleagues ${ }^{11}$ compared OW parents to SFR parents using the Nurse-Parent Support Tool. Significant differences were found between OW and SFR parents on three items. Parents in the OW felt that they were better informed about changes in their infants' conditions, that staffs were better at teaching them about how to comfort their infants, and that they were more optimistic about their infants' health. It is interesting to note that the other 19 items assessing support did not show significant differences.

In contrast, the Carter and colleagues ${ }^{15}$ study surveyed parents whose infants were moved from an OW to new SFRs. They found that after this transition parents felt more supported, informed, and perceived significant improvements in their access to nurses and physicians. Parents also felt they had more privacy and were able to spend more time with their infants. A potential confounder in this study was the change in visitation policy that occurred with the opening of the new SFR unit. The old OW had limited parental visitation hours whereas the SFRs had unlimited visitation hours. Rather than the design of the unit, it is possible that parents' increased perception of support may have been in part due to prolonged time spent at bedside, resulting in better access to staff and support. Given the limitations of existing studies, further investigation was required to expand our understanding of support for parents in SFR NICUs.

The aim of this study was to explore the support experience of parents, that is, the experiences of mothers and fathers in SFRs of the NICU. Understanding parents' experiences of support during their stay could help to inform health care providers on how best to support parents in SFRs. The research question guiding this study was: What is parents' experiences of support in SFRs in the NICU?

\section{METHODS}

\section{Design}

A qualitative descriptive design was chosen for this study because no exploratory descriptive work existed on the topic of parents' experiences of support in SFR NICUs. It allows for the description of parents' perceptions using the language of the participants themselves. ${ }^{25}$ The intention was to generate a straightforward, richly detailed description of participants' perspectives to provide an in-depth understanding of their NICU experiences. ${ }^{25,26}$

\section{Setting and Participants}

Participants were recruited from a Canadian Level III NICU, with 40 beds, eight of which are SFRs. These eight rooms, known as Family Integrated Care Area (FICA), are situated apart from the rest of NICU, which consists of five 6-bed pods and two isolation rooms. Infants who require acute medical care are first admitted to pods. As they grow 
and stabilize, they are moved into FICA, where families are prepared for discharge. In the FICA area of this unit, the nurse-to-infant staffing ratio is 1:4. Participants were recruited from July 2017 to May 2018. The target sample size was $15-20$ parents, which is deemed appropriate for qualitative descriptive design. ${ }^{26}$

The participants were the mothers and fathers of NICU infants in FICA, since this study aimed to understand the experience of both parents in SFRs. Parents were eligible for inclusion if (1) they were visiting their infant during his/her stay in FICA; (2) they were able to speak and read English or French; (3) they were over the age of 18 ; (4) their infant had been hospitalized in FICA for more than 48 hours regardless of gestational age, birth weight, or reason for admission; and (5) their infant was expected to be in FICA for at least another 48 hours. While both parents of an infant could consent to participate, this was not required. Parents were excluded if their infant ( 1 ) had a diagnosis of Grade 4 intraventricular hemorrhage; (2) had trisomy 21 or other chromosomal abnormalities; (3) was in palliative care; and/or (4) was being placed in foster care. The exclusion criteria addressed families whose infants may have had special needs.

\section{Procedure, Analysis, and Trustworthiness}

Eligible parents were approached by designated NICU nurses who assisted with recruitment, and only those parents who agreed to be informed about the study were contacted by the research team. At the time of enrollment, information regarding the study were given to parents, and informed written consent was obtained along with parent demographic information and infant medical data. Data were collected using smartphones preinstalled with an application called Handy Application to Promote Preterm infant happY-life (HAPPY), which was developed by a member of the research team. Smartphones were loaned to participants to record their experiences in real time to minimize recall bias. Past NICU studies used journaling to collect data from participants, but this led to a high level of attrition, as parents felt that journaling took away from their time with their infant. ${ }^{10}$ The HAPPY application had been tested in previous NICU studies with parents and nurses in Canada and in Finland, and was found to be a feasible and acceptable way for participants to share their experiences without being burdened. ${ }^{27-29}$

Each participant had access to the smartphone to record their experiences for a 48 -hour period of time, which enabled them to record both in hospital and at home. During that time, parents were asked to describe their thoughts and feelings for when they felt supported or nonsupported, by opening the HAPPY application and choosing one of two buttons: "Support" or "Nonsupport." Parents then spoke into the smartphone describing their experiences. Parents were asked to include as much detail as possible. If both parents of a single family wished to participate, they made independent recordings to avoid influencing each other. At the end of the
48 hours, the smartphones were collected by the research team. Recordings were extracted and deleted from the smartphones and stored on a research computer. All recordings were then transcribed verbatim in the language in which they were recorded.

Data collection and analysis occurred simultaneously, and the data were analyzed using content analysis, which is defined as a process of analyzing and integrating qualitative data according to prominent themes or concepts. ${ }^{30,31}$ Transcripts were read repeatedly by the research team, who are the authors of the present study including two nurse researchers, a nurse PhD student, and a nursing master's student. The first five transcripts were open-coded independently by every member to assess whether the same ideas and interpretations were drawn from the transcripts. This also ensured a thorough examination of the data. Subsequently, transcripts were open-coded (coded line-by-line) using the software N-Vivo. ${ }^{30}$ Similar codes were grouped together and labeled with unique descriptors. A back-and-forth process between the transcripts and the products of analysis facilitated the clustering of codes into subcategories and larger categories that captured parents' experiences of support. Weekly iterative discussions helped the research team to continuously examine the relationships and patterns in the data. Concurrent data collection and analysis allowed for new ideas to be generated and reviewed to inform subsequent analysis. ${ }^{32}$ This also helped to determine saturation, which was achieved after the eleventh participant, when no new ideas emerged from subsequent participants.

The research team took measures to ensure trustworthiness, a term which refers to whether qualitative findings are credible, transferable, dependable, and confirmable. ${ }^{33}$ Credibility, defined as the confidence in the truth of the data and its congruence to reality, ${ }^{33}$ was promoted by frequent debriefing sessions and by minimizing researcher bias through researcher triangulation, which is the reviewing of findings by multiple analysts in order to minimize blind spots in the analysis. ${ }^{33}$ For the reader to judge the transferability of our findings, we have provided sufficient contextual information about the participants and the study setting, as well as a rich description of the phenomenon under investigation. Dependability, known as the stability of data over time, ${ }^{33}$ was ensured using an audit trail-a detailed account of the decisions made during data collection and analysis-that was kept for the duration of the study. Lastly, confirmability, defined as the congruence between two or more individuals about the accuracy of the interpretation, was ensured using an audit trail and researcher triangulation. ${ }^{33}$

\section{Ethical Considerations}

Ethics approval was obtained from the institution where the study was conducted. There were no conflicts of interest. Written and informed consent was obtained from all participants. 


\section{RESULTS}

\section{Participants}

The participants ( $n=15$, one couple) were nine mothers and six fathers. Ten (66.7 percent) of the participants were first-time parents. Mothers visited on average 9.8 hours per day $(S D=6.8)$, while fathers visited 5.1 hours per day $(S D=$ 3.6). The participants were predominately immigrants, with only five (33.3 percent) born in Canada. Infants $(n=14)$ were born at a gestational age between $24+6$ and $35+4$ weeks. Twelve ( 85.7 percent) were singletons and two ( 14.3 percent) were twins. See Tables 1 and 2 for further information on parent and infant characteristics.

\section{Description of Events Recorded}

Participants recorded a total of 64 events $(M=4.3, S D=$ 2.2). Forty-two (65.6 percent) events were classified by participants as support $(M=2.8, S D=1.5)$. Twenty-two (34.4 percent) of the recorded events were classified as nonsupportive $(M=1.5, S D=1.1)$. Mothers recorded 42 (65.6 percent) events, while fathers recorded 22 (34.4 percent) events. 64.3 percent of mothers' recordings were support, while 68.2 percent of fathers' recordings were support. Of all recorded events regarding personnel, 68.0 percent were about nursing staff, 18.0 percent about physicians, 6.0 percent about other

TABLE 1 Parent Demographic Characteristics

\begin{tabular}{|c|c|c|c|c|}
\hline \multirow[b]{2}{*}{ Characteristics } & \multicolumn{2}{|c|}{ Mothers $(n=9)$} & \multicolumn{2}{|c|}{ Fathers $(n=6)$} \\
\hline & $n$ & $\%$ & $n$ & $\%$ \\
\hline \multicolumn{5}{|l|}{ Marital status } \\
\hline Single & 0 & 0.0 & 0 & 0.0 \\
\hline Cohabiting or married & 9 & 100.0 & 6 & 100.0 \\
\hline \multicolumn{5}{|l|}{ Education level } \\
\hline Bachelor or higher & 7 & 77.8 & 2 & 33.3 \\
\hline Junior college & 2 & 22.2 & 3 & 50.0 \\
\hline High school & 0 & 0.0 & 1 & 16.7 \\
\hline \multicolumn{5}{|l|}{ Citizenship status } \\
\hline Born in Canada & 2 & 22.2 & 3 & 50.0 \\
\hline Naturalized citizen & 2 & 22.2 & 1 & 16.7 \\
\hline Permanent resident & 4 & 44.4 & 1 & 16.7 \\
\hline Other & 1 & 11.1 & 1 & 16.7 \\
\hline \multicolumn{5}{|l|}{ Employment status } \\
\hline Employed & 2 & 22.2 & 5 & 83.3 \\
\hline Parental leave & 3 & 33.3 & 0 & 0.0 \\
\hline Student & 1 & 11.1 & 0 & 0.0 \\
\hline \multirow[t]{2}{*}{ Unemployed } & 3 & 33.3 & 1 & 16.7 \\
\hline & $M$ & $S D$ & $M$ & $S D$ \\
\hline Age & 34.6 & 5.1 & 35.0 & 8.1 \\
\hline Hours spent in SFR daily & 9.8 & 6.8 & 5.1 & 3.6 \\
\hline
\end{tabular}

Abbreviation: SFR = single-family room.
TABLE $2 \square$ Infant Characteristics

\begin{tabular}{|c|c|c|c|}
\hline \multirow[b]{2}{*}{ Characteristics } & \multicolumn{3}{|c|}{ Infants $(n=14)$} \\
\hline & $n$ & & $\%$ \\
\hline \multicolumn{4}{|l|}{ Sex } \\
\hline Male & 9 & & 64.3 \\
\hline Female & 5 & & 35.7 \\
\hline \multicolumn{4}{|l|}{ Parity } \\
\hline Single birth & 12 & & 85.7 \\
\hline \multirow[t]{2}{*}{ Twins } & 2 & & 14.3 \\
\hline & $M$ & $S D$ & Range \\
\hline Gestational age at birth (wk) & 30.6 & 3.3 & $24.6-35.4$ \\
\hline Birth weight (g) & 1584.0 & 734.1 & $620-3,605$ \\
\hline Days of life at recruitment $(d)$ & 37.7 & 37.2 & $5-108$ \\
\hline
\end{tabular}

parents, 4.0 percent about technicians, and 2.0 percent about lactation consultants and clerical staff, respectively.

Compared to recorded events reported in a previous HAPPY study ${ }^{29}$ conducted with NICU parents in which 20 parents recorded 133 events $(M=6.7, S D=3.3)$, parents in the present study recorded fewer events overall and on average. This may be because previous participants were recruited during all stages of hospitalization (e.g., intensive, intermediate, or step-down care), while current participants were only recruited from step-down. Thus, parents may have different priorities prior to discharge, which may affect their time and ability to record.

\section{Overview of Findings}

Parents described both support and nonsupport experiences, which are depicted in Figure 1. The center of the figure represents the recipients of supportive care, which could be the parents or the infants. Parents felt supported when care was directed toward them as well as when care was provided to the infant. How well staffs were able to care for the family as a unit played a significant role in parents' overall experience of support. The second layer represents the persons who provided support. While most events were related to the behaviors of nursing staff, other members of the health care team were also identified. The third layer consists of the first two major elements of support: care actions and how care is provided. These concentric layers are situated within the third major element of support, the SFR environment, which affected parents by directly contributing to their experiences of support or indirectly influencing the behavior of staff. The following sections describe each of these elements.

\section{Care Actions}

Coaching and Learning Collaboratively. Parents felt supported when staff guided them in their learning; for example, when nurses taught them how to address the physical needs of their infants, about infant health and development, or about 
FIGURE 1 Concept map of support.

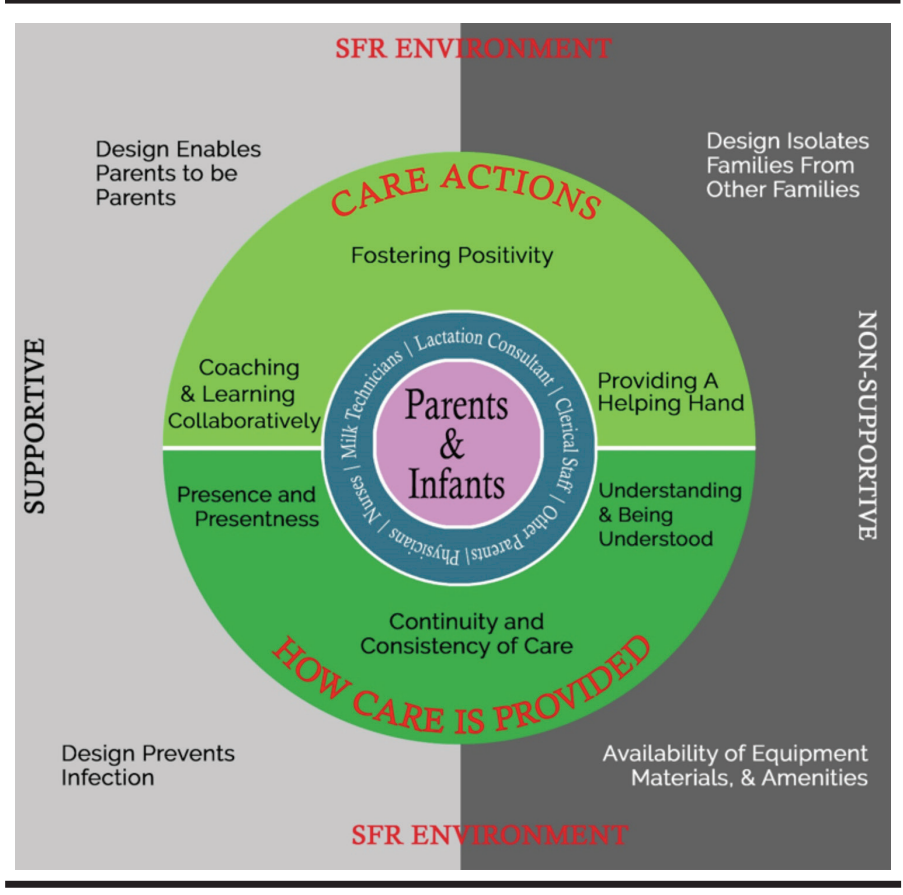

Abbreviation: SFR = single-family room.

Note. This concept map summarizes all of the major ideas rising from analysis. At the center of the concept map is the recipient of care. The second layer comprises the people who gave support. Three major elements influence parents' experiences of support: actions of care, how these actions are provided, and the SFR environment-the overarching context within which care is given.

the health care system. Parents reported support when nurses provided verbal instruction, demonstrated care techniques, and coached parents during hands-on caregiving. Parents felt particularly supported when staffs were sensitive to their level of comfort and worked collaboratively with the parents to meet their learning goals. As one mother noted, "What I appreciate about the nurses here [is that they] make you [become] autonomous [in] the good kind of way. [...] They don't force you into it. First, they show you how to do it, and if you're comfortable doing it, they'll supervise you, but then after a while, they'll let you go and fly on your own" (001MT).

In contrast, when parents' comfort levels were not recognized, and their learning needs not addressed, parents felt nonsupported. Parents of preterm infants may spend many weeks, even months in the acute care pod area of the NICU before being transferred to a SFR for step-down care. Some parents described having a lack of information once they arrived in the SFR. They perceived a discrepancy between how comfortable they were caring for their infant independently and how comfortable the staff believed they were: "It felt really as if [the nurses thought] I was already comfortable . . because I've already been here for three months with my baby" $(011 \mathrm{MT})$. This suggests that staffs were sometimes unaware of parents' learning needs. These parents felt unready for the level of autonomy that some nurses expected of them.
Fostering Positivity. Parents felt supported when nurses helped sustain a positive perspective on their infants and their caregiving abilities. When infant caregiving became challenging, parents reported that nurses' positive attitudes helped them "keep the faith" and maintain their optimism. Verbal encouragement helped them persist in the face of challenges. One parent noted, "[The nurse] was cheering me up a bit because this time the [breastfeeding] didn't go as well as before. So, she was really supporting me, encouraging me not to give up" (001MT). Additionally, parents experienced support when staff provided them with positive feedback concerning caregiving tasks they were able to undertake. One mother recounted how she could as a first-time parent confidently perform new caregiving skills because the nurse had commended her for "doing it well" (008MT). Through fostering positivity, nurses promoted parents' morale, built their self-confidence, and highlighted for them their growth as caregivers. Parents did not record any nonsupport experiences with regard to fostering positivity.

Providing a Helping Hand. Parents felt supported when staff assisted with caregiving or assumed infant caregiving tasks on parents' behalf. Often, parents could provide infant care by themselves with some assistance. In these cases, they found it supportive when staff helped manipulate equipment or fetch supplies. In contrast, when parents did not feel comfortable doing tasks themselves or when they were unavailable due to competing responsibilities, they felt supported when nurses assumed caregiving. Many parents appreciated being able to step back or focus on other things from time to time by taking a break, spending time with their partner, or taking care of their family at home. When nurses anticipated parents' needs in advance and informed them that assistance was readily available if they required it, parents felt supported. One parent (009MT) stated, "I can do the bath, but [the nurse] still made sure I was comfortable, if I ever need help or anything, she was available to help do the bath." Support for parents meant that parents cared for their infants to the extent they were comfortable but nursing staff stepped in whenever parents could not fulfill the caregiving role. Parents did not report any nonsupport experiences for this category.

\section{How Care Is Provided}

Presence and Presentness. Parents experienced support when staff was present and available to them in the SFRs. They described staff "being present" on two distinct levels. First, parents felt supported when they could have direct contact and dialog with staff. Many parents perceived supportive nursing as nurses being physically available to address parents' questions and concerns: "When we had questions, nurses were there" (003MT). However, when nurses were not as present as parents expected them to be in the SFRs, parents felt nonsupported. One parent spoke of her insecurities being in a SFR by herself when she did not know where the nurses were and she wanted them to "check up on parents, pop in, 
making sure parents are okay" (010MT). However, she did not fault the nurses for being less present, realizing that it was because of nurse-to-infant ratio changes in the SFR environment, which indirectly affected nursing behavior. Parents also perceived a lack of medical staff's presence as nonsupportive. Compared to the pods where parents reported seeing doctors "all the time" during rounds, parents stated that they rarely saw doctors in SFRs, even though medical rounds were also conducted in this area of the NICU. This lack of physician presence was considered nonsupportive if the room door was closed and medical staff did not enter the room: "They stay in the corridor, so I don't see them" (011MT). The lack of face-to-face contact with physicians left parents feeling "less aware" of their infants' health status and less supported by the medical team compared to what they experienced in the pods.

In addition to staff's physical presence, parents described support as staff being attentive in the moment; that is, they dedicated significant uninterrupted time to provide care and that their attention was not divided. This quality was particularly important for nurses, as they were typically the personnel assisting families with their needs. In contrast, parents experienced nonsupport when nurses could not dedicate time to the family, but were instead distracted, preoccupied or rushing when providing care. "The nurse was [...] rushing. [...] It's not [an] ideal situation where nurses don't have enough time to take care of each [baby]" (010MT). Parents explained that when care was expedited, they felt overwhelmed, uncertain, and not supported.

Continuity and Consistency of Care. Parents felt supported when the same nurse cared for the family over time. When there were changes in nurse assignment, parents described support as receiving consistent care and information across nurses. To some, the continuity and consistency of care helped them understand "the evolution" of their infants' health. Parents repeatedly used phrases such as, "always," "every time," "during my whole stay," suggesting that consistency of nursing care across time was perceived as an important aspect of supportive care. When nurses provided continuous and consistent care, parents felt better supported and perceived staff as more knowledgeable and competent, which in turn fostered a sense of trust and security between the parent and the care team.

Conversely, when parents did not experience continuity or consistency of care, they felt nonsupported. Parents felt nonsupported when nurses not previously known to them assumed care of the infant, as well as when there were discrepancies and inconsistencies in information and care practices. Parents felt frustrated and confused by inconsistencies and did not know which source to trust. Often, these inconsistencies meant that parents' questions and concerns were not adequately addressed. When discontinuity of care occurred in tandem with inconsistencies of care following a change in nursing staff, parents worried that staff lacked the competency to care for their infants. "All the other nurses told me that suctioning is required during every care. [This nurse] didn't know the baby, she didn't know the baby's history, that's why she didn't suction" (006MT). Discontinuity and inconsistencies deterred parents from forming trusting relationships with staff, leaving them feeling uncertain and nonsupported.

Interestingly, one father had a distinct perspective: he appreciated differences in certain care practices. Unlike others, he felt supported by the "various techniques and approaches of different nurses" (013FT). For him variations demonstrated that caring for his infant "[was] not an exact science," which allowed him to test out and select the best technique for his infant.

Understanding and Being Understood. Parents reported feeling supported when they could understand the information being provided, and had their own opinions and voices acknowledged and considered by the health care team. Parents experienced support when both medical and nursing staff offered clarification in a way that was comprehensible and had meaning for parents: "I had an answer and not only an answer, but I had an explanation and [the nurse] really ensured that I understood" (001MT). Communication was supportive when staff listened to parents' concerns and acted on these. For instance, one mother described how a nurse supported her by facilitating dialog between the parent and the neonatologist to address the mother's concerns regarding her infant's health. Parents also experienced support when staff maintained a nonjudgmental attitude. "[The nurse] understood [. . . ] if I wasn't able to participate in the care, she didn't judge me as a parent" (010MT). Moreover, parents felt understood when staff related to them as fellow parents. When nurses could "get" parental struggles, parents described feeling well supported.

In contrast, parents felt nonsupported when they did not understand decisions made by the care team and/or when their opinions were not considered in decision making. They felt nonsupported when care plans "would be modified seemingly without any explanation" to the family, or when the medical team made decisions without including "the first-hand accounts of nurses and parents" (013FT). The exclusion of parents from decision making elicited confusion, frustration, and a sense of disconnection. This led parents to believe their opinions were not valued, which resulted in their feeling nonsupported. Effective support meant parents understanding staff perspectives, but also staff understanding parents' perspectives.

\section{The SFR Environment}

Design Enables Parents to Be Parents. Many parents felt supported by the SFR design. They considered the privacy supportive as it provided a space where they could learn and practice caregiving skills with the help of the staff but without the scrutiny of an audience: "Even when we pumped milk, we didn't need a curtain to hide behind, there aren't many people, we are alone, and we are free to pump milk without 
embarrassment, without people seeing us" (006MT). Parents emphasized that in the SFR they did not feel judged or observed, so that they were able to learn new skills confidently. Additionally, the autonomy that parents experienced in SFRs was viewed as supportive. As one father noted, "Most of the time, we are really autonomous in the way that we take care of the baby" (018FT). He went on to explain that when they were able to independently care for their infant this underscored their capability to care for their infant at home and contributed to their readiness for discharge.

Furthermore, parents felt supported by the family-friendly environment which allowed them to engage in parenting activities beyond basic caregiving. Parents considered SFRs more amenable to attachment with their infants: "FICA allowed us to be more comfortable as parents, so we can do things like sing, dance, listen to music" (013FT). The SFR design was often described as "making it easier for [parents] to be present" by their infants' bedsides and to rest.

Design Prevents Infection. Parents felt supported by SFR's capacity to prevent the spread of infection. They indicated that SFRs served as a physical barrier between the family and carriers of infectious disease, such as staff, other patients, parents, and visiting families. Moreover, parents appreciated the individualized equipment in their room: "[Families] have their own fridge, own materials that they need, especially the warming of the milk, [their] own chair [. . .] the pumping machine. There's no sharing" (008MT). Parents believed that the SFR design protected their infants against infections, leading parents to feel safe and supported.

Design Isolates Families from Other Families. While some parents reported feeling isolated from other families, others did not. One mother, for example, described feeling unsupported because of the isolation she felt from other parents: "However, in a [pod] we have other parents, often we can ask, everyone talks about their experience and it comforts the parents" (006MT). Compared to when her infant was in a pod, this parent experienced the limited access to her peers in the SFR as nonsupportive. Nevertheless, another mother $(003 \mathrm{MT})$ described being able to interact with parents in SFRs, suggesting no difference between the two settings. One father $(018 \mathrm{FT})$ described feeling more isolated from other families in SFRs; however, he explained that he did not perceive this isolation as necessarily nonsupportive: "But like I was saying, on the other hand, we still need to isolate ourselves a little bit and find ourselves again, so it is not entirely negative when we're there." While there were fewer other families with whom he could interact, he considered this as a normal part of discharge and going home.

Availability of Equipment, Materials, and Amenities. Parents felt nonsupported when they did not have sufficient material or equipment to facilitate their activities in SFRs. When materials or equipment were not available, parents reported being frustrated and less supported. Missing equipment was disruptive, adding to the stress parents may already be experiencing during caregiving. They also felt nonsupported when the lack of amenities, such as lack of beds for both parents to sleep overnight, hindered their remaining at their infants' bedsides. As one father stated (017FT), "It would be cool to have two sofa beds. Like ... [dad] is not obliged ... he can stay with the mom, and then he does not have to stay in the same sofa."

\section{Differences Between Mothers and Fathers}

While both mothers and fathers recorded about all three major elements of support, there was a difference in emphasis between mothers and fathers. More mothers described care actions compared to fathers. In contrast, fathers were concerned with support as it related to the SFR environment, design, and equipment. However, both mothers and fathers were equally concerned with the presence and presentness of staff. All parents except two (one mother, one father) recorded a supportive or nonsupportive experience concerning staff presence or presentness.

\section{DISCUSSION}

Parents' support experiences in the SFRs were influenced by three overarching elements. The first two elements were the care actions of staff and how care was provided. The third element, the SFR design, shaped support either by directly affecting parents' day-to-day infant caregiving activities or indirectly by influencing the behavior of staff who must provide care in the SFR. Past descriptions of support for NICU parents ${ }^{24}$ have not differentiated the support needs of parents in different unit environments and at different levels of infant acuity. Our findings contribute to our understanding of support for parents in step-down SFRs. The four components of nursing support for parents of hospitalized infants described by Miles and colleagues ${ }^{24}$ (i.e., supportive communication and ongoing information, maintaining the parental role, providing emotional support, and providing care to the infant) are all evident in our findings. Our participants experienced support in SFRs as having access to a team that informed parents about their infants' health and included parents in decision making, as well as having an attentive nurse who coached parents collaboratively and fostered a positive view on their situation and caregiving.

Consistent with previous literature, parents in the present study noted that SFR design enabled privacy. Previous studies ${ }^{5,11,15}$ have reported better privacy for families in SFRs NICUs as compared to OW. Parents in our study felt more comfortable behaving as a family in SFRs, noting how private rooms encouraged their presence and made it both easier and more comfortable for them to be at the bedside. As in previous studies $^{34,35}$ that found both mothers and fathers appreciated not being observed by others, our participants emphasized the importance of having a space that enabled them to be parents without being disturbed and without disturbing others. 
Another critical benefit of this privacy that was particularly supportive was how the SFR provided a safe space for staff to teach parents, and where parents could learn and practice new skills and techniques without being observed. When parents are learning in circumstances where they can be seen by others, NICU nurses should aim to maximize privacy to the greatest extent possible through practices such as pulling curtains, closing doors, and knocking before entering the private rooms.

Our findings also have important clinical implications for how nursing staff can support parental learning. Parents in the present study described supportive learning as nurses coaching them in a collaborative fashion while fostering positivity. Self-efficacy is an individual's belief of being able to manage a task effectively and successfully. ${ }^{36}$ De Montigny and Laicharite defined parental self-efficacy as "the beliefs or judgments parents hold of their capabilities to organize and execute a set of tasks related to parenting an infant," ${ }^{77}$ which can be influenced by four sources: (1) past performances (e.g., practicing a skill); (2) vicarious learning/ modeling (e.g., nurse demonstrating bathing); (3) verbal appraisal and feedback (e.g., commending parents); (4) emotional/physiologic response to a task (e.g., nurse being positive and empathetic when parents could not perform a care activity). ${ }^{37,38}$ We found that nursing care actions such as collaborative coaching and fostering positivity addressed these sources of parental self-efficacy. When coaching parents through caregiving tasks, nurses offered parents the opportunity to practice and familiarize themselves with new skills at their own pace, all the while providing supportive supervision at their side. For new techniques that parents had not previously attempted, nurses helped parents learn through expert demonstrations. Parents also identified nurses acknowledging parents' efforts and commending them for doing well as supportive. Lastly, nurses supported parents who encountered difficulties mastering new skills. A combination of these supportive actions helped parents to gain confidence and be more autonomous and comfortable as independent caregivers. However, staff should be mindful of how much responsibility parents are asked to take on. In this study, parents demonstrated appreciation for when nurses carried out caregiving tasks and parents only did what they could. Evidence shows that overreliance on parents of pediatric patients may result in parents feeling neglected, stressed, and unsupported. ${ }^{39}$

Parents in the current study considered the SFR design supportive, in that it was comfortable for them to be at the bedsides of their infants, even overnight if they chose to do so. We observed that compared to a previous study by Treherne and colleagues, ${ }^{29}$ which was conducted at the same hospital but in the former OW unit, parent presence was higher in the current study $(9.8$ and $5.1 \mathrm{~h}$ on average per day for mothers and fathers respectively, compared to 5.4 and $4.1 \mathrm{~h}$ in the old unit). This observation is consistent with the study by Raiskila and colleagues, ${ }^{40}$ which found NICUs that facilitated overnight stays had greater parental presence compared to units without such facilities. Future design of SFRs should consider including resting areas and amenities that accommodate two parents.

Contrary to the findings of Carter and colleagues ${ }^{15}$ some parents in our study perceived a lack of access to nurses in the SFRs. As noted earlier, at the site where this study took place the SFRs are used to transition families from higher acuity pods to step-down care in preparation for transitioning home. This lack of access was perceived by parents as unsupportive in a critical period during which parents solidified their caregiving skills. Although lack of access could be attributed to the SFR design, this finding may also reflect the simultaneous change in the nurse-to-infant ratio that occurs at the study site because of the change in the level of acuity. The nurse to infant ratio is $1: 2$ in the pods, while it is $1: 4$ in the SFRs. SFR nurses should be aware that they may be perceived as unavailable to parents and should therefore take initiative to inform parents where staff may be found if they are not immediately within line of sight. Nurses should also encourage parents to feel comfortable seeking help when needed.

Parents of the current study differed in their perceived access to peer support. Some felt isolated, some did not, while some felt that isolation is needed for them to gain independence. Further investigation is needed to examine peer support for parents in SFRs, and specifically, the relevance of peer support for parents during various stages of their infants' hospitalization.

Transition points are known to be a time of stress and heightened need for NICU parents. ${ }^{41}$ Parents described support as consistency in care and information. Previous evidence indicates that inconsistencies in information and care practices during transitional periods can result in distress and fear in NICU parents, who are often unprepared for the differences in care practices and in the organization of care (e.g., pod vs. FICA). ${ }^{4-43}$ Taken together, these findings speak to the importance of clear communication between parents and nurses. Certain care practices such as interventions (i.e., suctioning, parainfluenza protocol) should be standardized and consistent across staff, while other practices such as infant bathing might be more flexible and subject to variation. Our findings suggest that clinicians should preemptively discuss with parents the natural variations that may occur in some care activities in order to help manage parental stress.

It is known that inadequate information during transition to home can be anxiety-provoking for the parents. ${ }^{41}$ Another major contributor to parents' experience of nonsupport was limited access to medical staff in SFRs, resulting in parents feeling less informed and less involved in decision making. This finding differs from the Carter and colleagues ${ }^{15}$ study in which parents in SFRs reported better access to nurses and physicians but is consistent with Domanico and colleagues' study ${ }^{11}$ in which parents in SFRs felt less informed. It may be that unit design is not the only difference across studies, but how medical staff conduct rounds may also differ. Axelin and colleagues $^{44}$ described different approaches to communication between physicians and NICU parents during medical rounds. 
Some of our participants described what Axelin identified as "neonatologist-led" or "disconnected communication" during medical rounds. It may also be that physician behaviors during rounds in our study may reflect step-down care with less frequent presence and interaction with parents. Nonetheless, parents preferred what Axelin described as "collaborative communication and decision-making." This has important implications for practice, as it is known that being included in decision-making processes makes parents feel more in control and creates a sense of closeness with their infants. ${ }^{29}$ Further research is needed to study physicians' behavior in SFRs and in step-down care in order to improve support for NICU parents through providing a more inclusive decision-making process.

\section{Strengths and Limitations}

A significant strength of this study is the method of data collection. The HAPPY app was a convenient method for parents to record their thoughts and feelings in real time to minimize recall bias and capture the richness of their experience. One limitation of our study is that our NICU integrates two types of design, pods and SFRs. The experiences of our participants may be unique in that their perceptions of support in the SFRs may be informed by what they experienced previously in a pod. Not only does the design differ between pods and SFRs, but the acuity level also differs. SFRs in the present study are used for discharge preparation, which may influence the behavior of nursing and medical staff, thus affecting parents' experience of support. Given our limitations, it would be beneficial to repeat a study in a SFR-only NICU, along with a larger sample size. It is important to note that a substantial proportion of our study participants were not born in Canada, and thus their experiences in acute care hospitalization may differ from native born parents who are familiar with the Canadian health care system. Additionally, parents were asked to describe their experience of support, some aspects of which may not be specific to the SFR setting, but rather reflect parents' support needs within NICUs irrespective of design.

\section{CONCLUSION}

Parents' experiences of support in SFRs in the present study identified support as staff collaboratively coaching parents through their learning journey, fostering their positivity, and providing situational assistance when appropriate. Additionally, parents identified support as staff being present to them in the SFRs while providing consistent and continuous care. Parents further identified support as staff communicating so that parents may understand staff and for their own opinions and feelings to be understood by staff. Lastly, parents found that the SFR design supported them through establishing a private space for parents to learn without an audience, to be with their infants as a family, and to be safe from the spread of infections. However, some parents found that the equipment and materials they needed to care for their infants may not be readily available in their private rooms, and one parent found the isolation from other parents to be nonsupportive.
Understanding parents' support needs is crucial in helping clinicians better care for families, so that parents may be well supported in all aspects during their infants' hospitalization.

\section{ACKNOWLEDGMENTS}

Nancy Feeley is supported by a Senior Research Scholar Award from the Fonds de recherche du Québec-Santé (FRQS). We would like to thank Kaitlen Gattuso, Tara O'Reilly, and Felicia Brochu for their assistance with data collection; Stephanie Lopresti for her assistance with data transcription; Nancy Vincelli and Stephanie Treherne and all nursing staff at Jewish General Hospital NICU who facilitated participant recruitment.

\section{REFERENCES}

1. Shahheidari M, Homer C. Impact of the design of neonatal intensive care units on neonates, staff, and families: a systematic literature review. J Perinat Neonatal Nurs. 2012;26(3):260-266. doi:10.1097/ JPN.0b013e318261cald

2. Johnson BH, Abraham MR, Parrish RN. Designing the neonatal intensive care unit for optimal family involvement. Clin Perinatol. 2004;31(2):353-385. doi:10.1016/j.clp.2004.04.008

3. Urlich R, Zimring C, Quan X, Joseph A, Choudhary R. The role of the physical environment in the hospital of the 21st century. The Center for Health Design. 2004. https://www.healthdesign.org/. Accessed April 18, 2018.

4. Shepley M, Harris D, White R. Open-bay and single-family room neonatal intensive care units. Environ Behav. 2008;40(2):249-268. doi:10.1177/0013916507311551

5. Carlson B, Walsh S, Wergin T, Schwarzkopf K, Ecklund S. Challenges in design and transition to a private room model in the neonatal intensive care unit. Adv Neonatal Care. 2006;6(5):271-280. doi:10.1016/j.adnc. 2006.06.008

6. Mann NP, Haddow R, Stokes L, Goodley S, Rutter N. Effect of night and day on preterm infants in a newborn nursery: a randomized trial. $\mathrm{Br}$ Med J. 1986;293(6557):1265-1267. doi:10.1136/bmj.293.6557.1265

7. Graven SN, Bowen F Jr, Brooten D, et al. The high-risk infant environment. Part 1 . The role of the neonatal intensive care unit in the outcome of high-risk infants. J Perinatol. 1992;12(2):164-172.

8. Long JG, Lucey JF, Philip AG. Noise and hypoxemia in the intensive care nursery. Pediatrics. 1980;65(1):143-145.

9. White R, Whitman T. Design of ICUs. Pediatrics. 1992;89(6):1267.

10. Jones R, Jones L, Feary AM. The effects of single-family rooms on parenting behavior and maternal psychological factors. J Obstet Gynecol Neonatal Nurs. 2016;45(3):359-370. doi:10.1016/j.jogn.2015.12.015

11. Domanico R, Davis DK, Coleman F, Davis BO Jr. Documenting the NICU design dilemma: parent and staff perceptions of open ward versus single family room units. J Perinatol. 2010;30(5):343-351. doi:10.1038/jp.2009.195

12. Swanson JR, Peters C, Lee BH. NICU redesign from open ward to private room: a longitudinal study of parent and staff perceptions. $J$ Perinatol. 2013;33(6):466-469. doi:10.1038/jp.2012.157

13. Watson J, DeLand M, Gibbins S, MacMillan E, Robson K. Improvements in staff quality of work life and family satisfaction following the move to single-family room NICU design. Adv Neonatal Care. 2014;14(2):129136. doi:10.1097/ANC.0000000000000046

14. Van Enk RA, Steinberg F. Comparison of private room with multiple-bed ward neonatal intensive care unit environments. HERD. 2011;5(1):5263. doi:10.1177/193758671100500105 
15. Carter BS, Carter A, Bennett S. Families' views upon experiencing change in the neonatal intensive care unit environment: from the 'baby barn' to the private room. J Perinatol. 2008;28(12):827-829. doi:10.1038/ jp.2008.102

16. Domanico R, Davis DK, Coleman F, Davis BO. Documenting the NICU design dilemma: comparative patient progress in open-ward and single family room units. J Perinatol. 2011;31(4):281-288. doi:10.1038/jp.2010.120

17. Lester BM, Hawes K, Abar B, et al. Single-family room care and neurobehavioral and medical outcomes in preterm infants. Pediatrics. 2014;134(4):754-760. doi:10.1542/peds.2013-4252

18. Örtenstrand A, Westrup B, Broström EB, et al. The Stockholm neonatal family centered care study: effects on length of stay and infant morbidity. Pediatrics. 2010;125(2):278-85. doi:10.1542/peds.2009-1511

19. White RD. Individual rooms in the NICU—an evolving concept. $J$ Perinatol. 2003;23(S1):S22-S24. doi:10.1053/j.semperi.2010.10.002

20. Harris DD, Shepley MM, White RD, Kolberg KJS, Harrell JW. The impact of single family room design on patients and caregivers: executive summary. J Perinatol. 2006;26(S3):S38-S48. doi:10.1038/ sj.jp.7211583

21. White RD. The newborn intensive care unit environment of care: how we got here, where we're headed, and why. Semin Perinatol. $2011 ; 35(1): 2$ 7. doi:10.1053/j.semperi.2010.10.002

22. Pineda RG, Stransky KE, Rogers C, et al. The single-patient room in the NICU: maternal and family effects. J Perinatol. 2012;32(7):545-551. doi:10.1038/jp.2011.144

23. Erdeve O, Arsan S, Canpolat FE, et al. Does individual room implemented family-centered care contribute to mother-infant interaction in preterm deliveries necessitating neonatal intensive care unit hospitalization? $\mathrm{Am} \mathrm{J}$ Perinatol. 2009;26(2):159-164. doi:10.1055/s-0028-1095186

24. Miles MS, Carlson J, Brunssen S. The nurse parent support tool. JPediatr Nurs. 1999;14(1):44-50. doi:10.1016/S0882-5963(99)80059-1

25. Sandelowski M. Whatever happened to qualitative description? Res Nurs Health. 2000;23(4):334-340. doi:10.1002/1098-240X (200008) 23:4<334::AID-NUR9>3.0.CO;2-G

26. Magilvy JK, Thomas E. A first qualitative project: qualitative descriptive design for novice researchers. J Spec Pediatr Nurs. 2009;14(4):298-300. doi:10.1111/j.1744-6155.2009.00212.x

27. Feeley N, Genest C, Niela-Vilén H, Charbonneau L, Axelin A. Parents and nurses balancing parent-infant closeness and separation: a qualitative study of NICU nurses perceptions. BMC Pediatr. 2016;16(1):1-13. doi:10.1186/s12887-016-0663-1

28. Bujold M, Feeley N, Axelin A, Cinquino C. Expressing human milk in the NICU: coping mechanisms and challenges shape the complex experience of closeness and separation. Adv Neonatal Care. 2018;18(1):38-48. doi:10.1097/ANC.0000000000000455

29. Treherne SC, Feeley N, Charbonneau L, Axelin A. Parents' perspectives of closeness and separation with their preterm infants in the NICU. $J$ Obstet Gynecol Neonatal Nurs. 2017;46(5):737-747. doi:10.1016/j. jogn.2017.07.005

30. Vaismoradi M, Turunen H, Bondas T. Content analysis and thematic analysis: implications for conducting a qualitative descriptive study. Nurs Health Sci. 2014;15(3):398-405. doi:10.1111/nhs.12048

31. Loiselle CG, Profetto-McGrath J. Canadian Essentials of Nursing Research. 3rd ed. Philadelphia, PA: Wolters Kluwer Health/Lippincott Williams \& Wilkins; 2011.

32. Thorne S. Data analysis in qualitative research. Evid Based Nurs. 2000;3(3):68-70. doi:10.1136/ebn.3.3.68

33. Lincoln YS, Guba EG. Naturalistic Inquiry. Beverly Hills, CA: Sage Publications; 1985.

34. Værland IE, Vevatne K, Brinchmann BS. Fathers' experience of starting family life with an infant born prematurely due to mothers' severe illness. Sex Reprod Healthc. 2017;13:8-13. doi:10.1016/j.srhc.2017.05.002
35. Flacking R, Dykes F. 'Being in a womb' or 'playing musical chairs': the impact of place and space on infant feeding in NICUs. BMC Pregnancy Childbirth. 2013;13(179):1-11. doi:10.1186/1471-2393-13-179

36. Bandura A. Self-efficacy mechanism in human agency. Am Psychol. 1982;37(2):122-147. doi:10.1037/0003-066X.37.2.122

37. MontignyF, Lacharité C. Perceived parental efficacy: conceptanalysis. J Adv Nurs. 2005;49(4):387-396. doi:10.1111/j.1365-2648.2004.03302.x

38. Bandura A. Self-efficacy: toward a unifying theory of behavioral change. PsycholRev. 1977;84(2):191-215.doi:10.1037/0033-295X.84.2.191

39. Coyne I. Families and health-care professionals' perspectives and expectations of family-centred care: hidden expectations and unclear roles. Health Expect. 2015;18(5):796-808. doi:10.1111/hex.12104

40. Raiskila S, Axelin A, Toome L, et al. Parents' presence and parent-infant closeness in 11 neonatal intensive care units in six European countries vary between and within the countries. Acta Paediatr. 2017;106(6):878888. doi:10.1111/apa.13798

41. Ballantyne M, Orava T, Bernardo S, McPherson AC, Church P, Fehlings D. Parents' early healthcare transition experiences with preterm and acutely ill infants: a scoping review. Child Care Health Dev. 2017;43(6):783-796. doi:10.1111/cch.12458

42. Franck LS, McNulty A, Alderdice F. The perinatal-neonatal care journey for parents of preterm infants: what is working and what can be improved. JPerinat Neonatal Nurs. 2017;31(3):244-255. doi:10.1097/ JPN.0000000000000273

43. Yang YY, He HG, Lee SY, Holroyd E, Shorey S, Koh SSL. Perceptions of parents with preterm infants hospitalized in Singaporean neonatal intensive care unit. J Perinat Neonatal Nurs. 2017;31(3):263-273. doi:10.1097/JPN.0000000000000239

44. Axelin A, Outinen J, Lainema K, Lehtonen L, Franck LS. Neonatologists can impede or support parents' participation in decision-making during medical rounds in neonatal intensive care units. Acta Paediatr. 2018;107:2100-2108. doi:10.1111/apa.14386

\section{About the Authors}

Lisa Xiaoyang $\operatorname{Liu}, \operatorname{MSc}(A)$, has a master's degree in nursing from McGill University. Currently, she is a first year medical student at Schulich School of Medicine and Dentistry, Western University.

Maryam Mozafarinia, RN, MSc, is a neonatal nurse with almost 13 years of clinical experience. She is also a PhD student at McGill University with her primary research focus being on premature infants and breastfeeding. Also, Maryam is a cofounder of a Canadian nonprofit and charitable organization "Une Chance de Vivre" with the primary goal of improving mothers' and premature infants' bealth and well-being in countries with limited health resources.

Anna Axelin, RN, PhD, is an associate professor at the University of Turku. She serves as the Director for Separation and Closeness Experiences in Neonatal Environment (SCENE) research group, which aims to improve parents' and infants' experiences and outcomes of neonatal care.

Nancy Feeley, RN, PhD, is an associate professor at McGill University's Ingram School of Nursing. She has an appointment as nurse scientist at Centre for Nursing Research and is also Project Director of Lady Davis Institute for Medical Research at Jewish General Hospital.

For further information, please contact:

Nancy Feeley, RN, PhD

Centre for Nursing Research Room H 301.1

Jewish General Hospital-McGill University

3755 Cote Ste Catherine Road

Montreal, Quebec, Canada H3T 1E2

E-mail: nancy.feeley@mcgill.ca 